\title{
BIM Electric Objects \\ Plug-in for Industry 4.0
}

\author{
Alberto Pavan, Andrea Cunico, Claudio Mirarchi, Dario Mocellin, \\ Elisa Sattanino and Valentina Napoleone
}

\begin{abstract}
The use of digital MEP objects in BIM models is a reality in the building world. This approach allows for the extrapolation of information relating to digital models, the simulation of their operation and the calculations of the sizing of plant circuits. The final goal of this research project is the creation of BIM object libraries for the 4.0 Industry, which includes the modelling of BIM objects for manufacturing connected with a company system. The Revit EasyBIM plug-in, developed for Vimar SpA and object of this research paper, allows for the creation of BIM objects, connected with a company system for the control of customer satisfaction throughout the production chain, design of use, installation, use in life cycle and disposal.
\end{abstract}

Keywords Building Information Modeling (BIM) • MEP electrical objects • Plug-in · Vimar SpA · Autodesk Revit

\section{Introduction}

The widespread presence of the BIM work methodology has involved several sectors of the world of constructions, one of which concerns products. Many manufacturers have invested, and still investing, on the digitization of their objects, in response to the increasingly widespread demand of designers to access their BIM libraries directly.

The digital object (very often referred to simply as "BIM Object") contains the technical characteristics of the product (geometry, performances, areas of use), but

A. Pavan $(\varangle) \cdot$ C. Mirarchi · E. Sattanino

Architecture, Built Environment and Construction Engineering-ABC Department, Politecnico di Milano, Milan, Italy

e-mail: alberto.pavan@polimi.it

A. Cunico · D. Mocellin

Vimar S.p.A., Marostica, Italy

V. Napoleone

Milan, Italy

(C) The Author(s) 2020

B. Daniotti et al. (eds.), Digital Transformation of the Design, Construction and Management Processes of the Built Environment, Research for Development, https://doi.org/10.1007/978-3-030-33570-0_7 
one of the main advantages is the fact that it is not only a mere container of information. Every BIM object, in order to be considered really as such, should have very specific requirements, ${ }^{1}$ such as the size in terms of $\mathrm{kB}$, the coherence of the informative attributes ${ }^{2}$ with the three-dimensional model, the level of detail differentiated according to the object LOD $^{3}$ scale, etc. (UNI11337).

Many of the BIM objects currently present and downloadable from the main BIM libraries are mostly objects that contain dimensional and informational data of the country in which they were modelled.

\section{The Project}

The research project "Study of modelling flows inherent in the production of BIM objects for MEP plant engineering", carried out in collaboration with Vimar s.p.a., has the goal of developing digital objects (MEP objects) according to the Italian market.

The need of designers (in particular plant engineers and architects) was analysed and, in the initial phase, it was decided to digitize the objects of the Vimar catalogue in order to create some models available for the users. After the first hypothesis of modelling the product, some criticalities emerged. The proposed strategy was to model the components in single objects: boxes, plates, and electrical modules (fruits) and then put them in a "container" family. The main disadvantage of this solution was the high weight of the "container" object (several MB), because in order to cover the whole range of possible combinations, it was necessary to nest all the fruit categories (sockets, control devices, data devices, etc.) to allow the end user to personalize the composition.

In addition, the digital object was difficult to use because the user had to manage parameters in order to choose both the $3 \mathrm{D}$ geometries and the $2 \mathrm{D}$ annotations to be displayed in the plant. To overcome this problem, it was decided to invest in the development of a Revit plug-in, with the aim of providing a real tool for the designer and not a simple preassembled library of digital objects.

In fact, the plug-in allows the user to instantly create the composition, ${ }^{4}$ choosing objects directly from the Vimar commercial catalogue.

\footnotetext{
${ }^{1}$ These requirements are illustrated in "BIM Object Standard NBS".

${ }^{2}$ Informational attribute means non-geometric information relative to a digital object (UNI 113371-2017).

${ }^{3}$ LOD means the level of development (or definition) of a digital BIM object (UNI 11337-1-2017).

${ }^{4} \mathrm{~A}$ composition means an object formed by a box, a plate, and one or more electrical modules.
} 


\section{Method}

Before reaching the decision to develop the plug-in, to understand the real needs of the reference market (designers, installers, companies, etc.) preliminary researches were conducted, through surveys and interviews with the main customers Vimar. From these first contacts, it emerged that the main software used by professionals was Autodesk Revit. Moreover, one of the needs that remained unresolved was to have "light" objects (file size of a few KB) that have 2D indications in a clear and well-readable plan in the different drawing scales.

Most of the currently modelled electrical objects have very detailed 3D geometry and a set of two-dimensional annotations nested within the object itself, which are managed through object parameters. To understand how to structure the workflow we analysed the catalogue of Vimar objects (divided into civil series, domotics, and video entry), their breakdown in categories according to their function and correlation with the respective 2D electric symbolism.

The categories identified are, for example, single-pole switch, bipolar switch, single-pole diverter, etc. This breakdown of the products made it possible to identify which informational attributes to link to the object and the geometry modeling strategy. The goal was to quantify the number of three-dimensional objects characterized by their own geometry, which differed according to the commercial series (Eikon, Plana, Arkè, Idea, and other series), to the number of modules (one, two, three, etc.) and to the specific function of the object (pushbutton, switch, electrical outlet, data socket, etc.). In parallel, the information attributes for each LOD scale were also analysed for the categories of electrical objects to be scanned.

The selection of the informative attributes involved the different scales of LOD, that is, American (BIMForum 2013), English (Pass1192-2 2013) and Italian (Norma UNI 11337).

Subsequently, two 3-dimensional prototypes were created with their informative attributes, evaluating the respective strengths and weaknesses of the different solutions.

The first object prototype, named "All in One", was modeled according to the modularity of the composition: then distinct objects were modeled based on the maximum number of devices you could insert. The object provided the possibility of manual choice of the 3D electrical modules to be displayed inside the box and the association of the corresponding 2D symbol displayed in the label. This label was modeled as a generic nested annotation within the "All in One" object (Figs. 1 and 2).

As you can see from the previous image, the user can select the electrical device to be displayed in "Position 1" and the corresponding 2D symbol to be displayed in the drawing label in "Position 12 D". Each single moulded device contained the respective electrical connector MEP.

The second object hypothesis (Fig. 3), called "Drag and Drop" provides the possibility of dragging the electrical symbols onto the label, allowing for more flexibility in the design. 
A. Pavan et al.
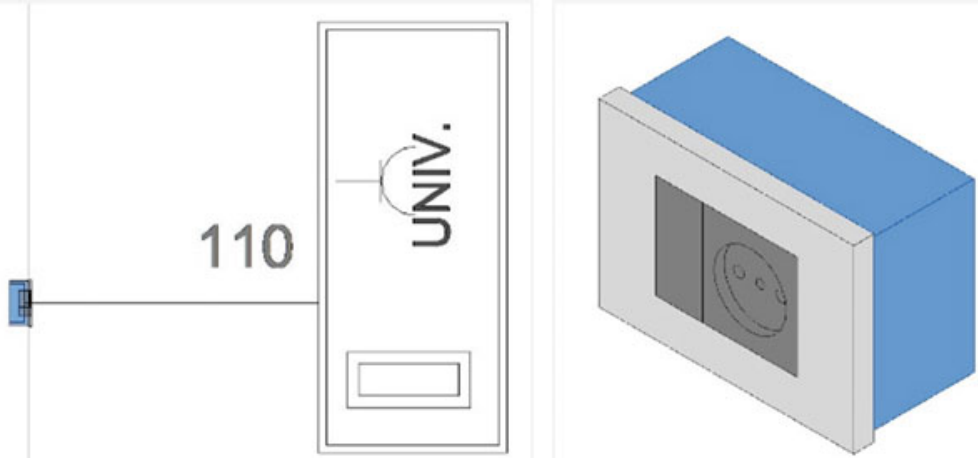

Fig. 1 Example of a plan view and a 3D view of an object, "All in One"

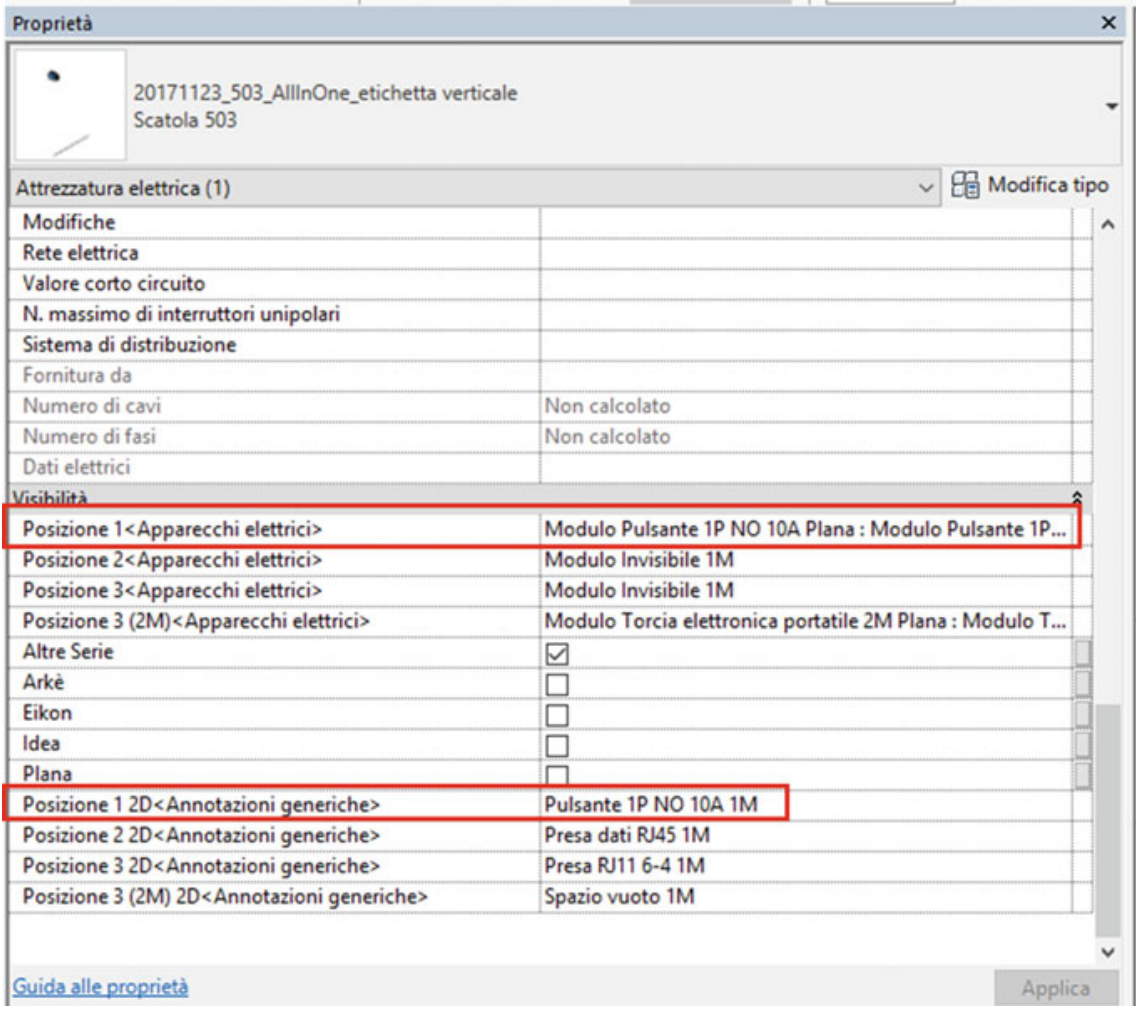

Fig. 2 Object properties window "All in One" 


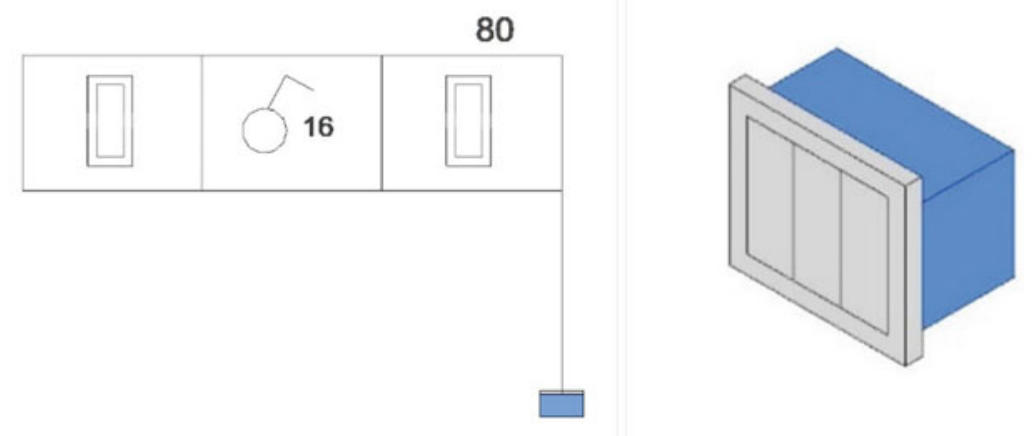

Fig. 3 Example of a plan view and 3D object, "Drag and Drop"

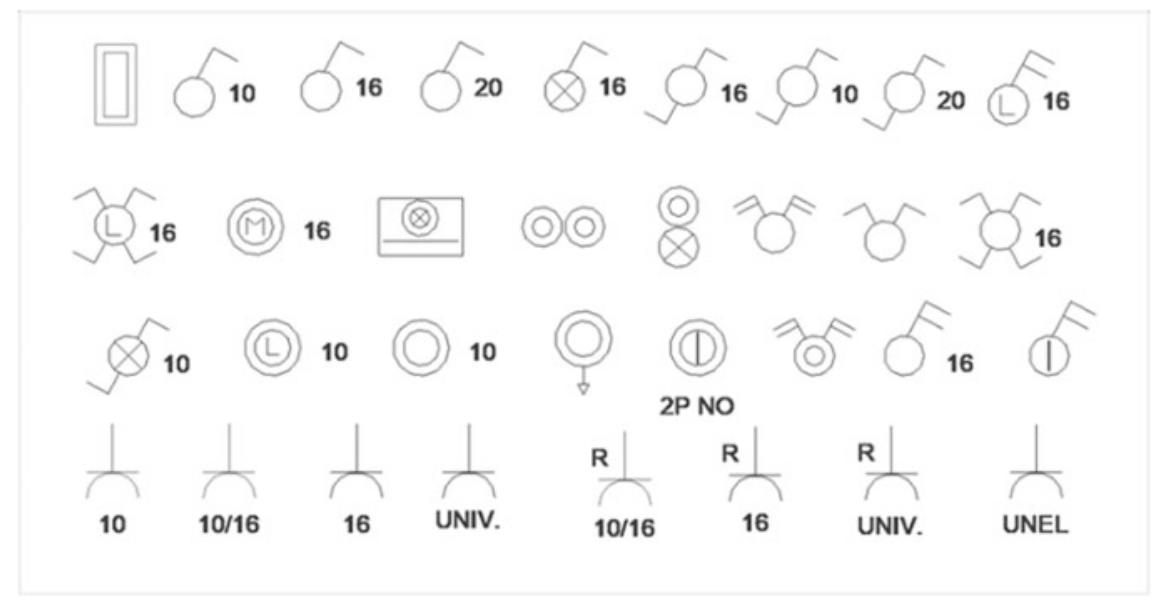

Fig. 4 List of electrical symbols object, "Drag and Drop"

The electric symbols (Fig. 4) to be dragged have been modeled as 2D annotations with the electrical connector MEP inside, in order to be inserted in a Revit electric circuit. These symbols, after being dragged and placed on the label surface (modeled as a 3D solid nested within the composition), were hooked to the host and then solidly placed in the object shifts. One of the drawbacks, however, of this solution was related to the real nature of the label modeled as solid, which in the case of clash detection could return false positives, ${ }^{5}$ as being a geometry visible in the space-could interfere with other objects in the model (e.g. Label/wall interference).

The label modeled in the two three-dimensional objects hypothesis shows the installation quota from the finished floor expressed in centimeters, automatically

\footnotetext{
${ }^{5}$ They mean positive results in the control of geometric interferences, but they do not mean a real problem of the object.
} 


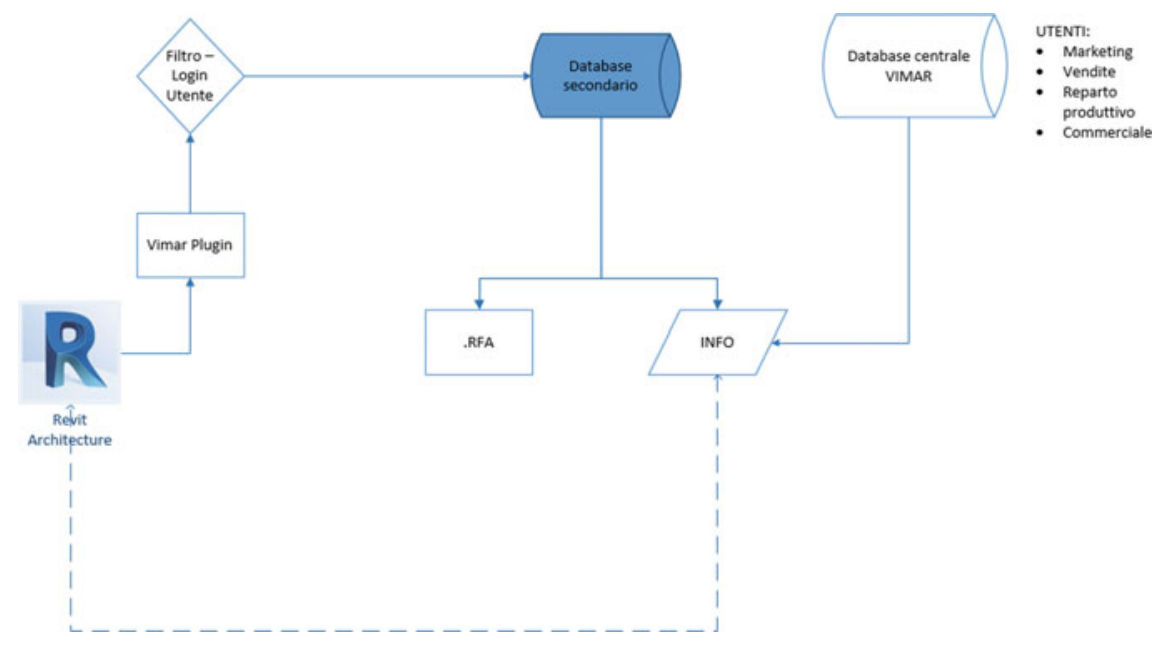

Fig. 5 Hypothesis workflow, Revit Plug-in

detected by the parameter set in the object. In addition, the shape of the label varies according to the choice of the commercial series (for example, the rectangular shape indicates the Eikon series).

Both of the proposed solutions have a serious disadvantage, which is the thousands of possible combinations (based on modularity, commercial series, and type of electrical modules). To realize a single object that can cover the entire case means making a very heavy object and therefore difficult to use.

To overcome this problem, a workflow was hypothesized to use a Revit plug-into manage both the composition of the three-dimensional electrical objects and the information relating to them (Fig. 5).

\section{Results}

The end result obtained from the research project was the creation of a Vimar plug-in (Fig. 6) able to automatically compose the three-dimensional object (consisting of a box, plate, and electric modules) according to the user's needs. It has been arranged into a graphical interface that is identified with the plug-in EasyBIM. ${ }^{6}$

Within the EasyBIM menu, there are several specific commands, grouped under "Compositions", "Logical Links", and "Separate".

\footnotetext{
${ }^{6}$ In order to download the plug-in from the Vimar website, it is necessary to register on the company's website and, once installed locally on the user's platform, it appears in the Revit taskbar under the EasyBIM tab.
} 


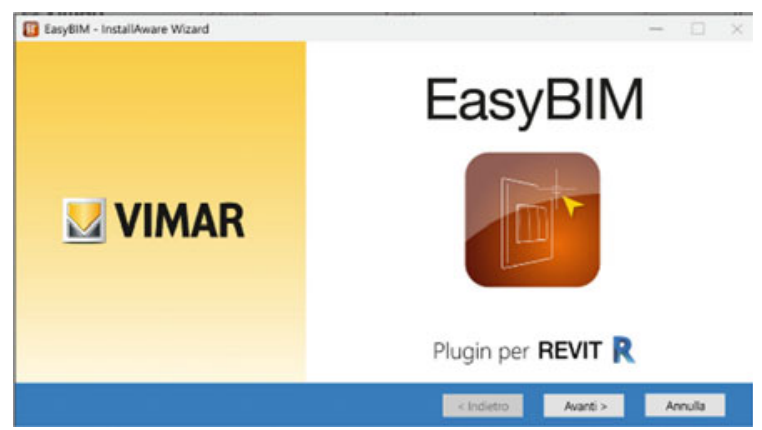

Fig. 6 EasyBIM plug-in for Revit

The main function of the Revit add-in is to be able to configure the electrical objects, choosing them from the Vimar price list or from a generic one. It was considered appropriate to give users the possibility to create objects that are "generici" (generic), or without make or model, to be used for public procurement or in preliminary/definitive projects, in which the commercial model of the product has not yet been chosen (Fig. 7).

In the graphical interface of the Configurator, there are some fields automatically managed by the plug-in (for example, N. Box) While the others are at the discretion of the user, such as "abbreviation" and "description". The "Elevation" field allows you to set the installation quota of the composition from the floor of the finished floor. In the dropdown menus below you can choose the price list (generic or Vimar), the commercial series (in the case of Vimar products the commercial series currently

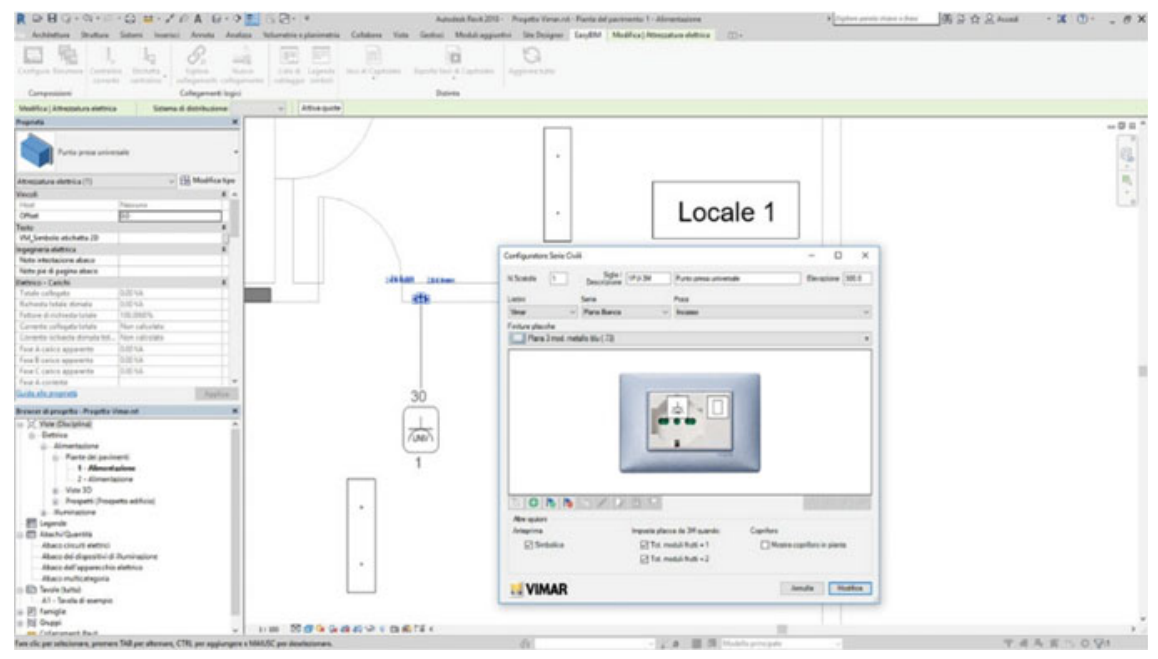

Fig. 7 Examples of graphical interfaces in the case of a Vimar object configuration 
available are Eikon, Arkè and Plana) and finally the type of laying (built in traditional walls, incasso in light walls and wall installation with IP protection degree). In the Configurator interface there is also a two-dimensional preview box that allows you to visualize the object you are composing and to modify the electrical modules through the commands in the bar. Through the use of these commands you can:add a new device; automatically add the hole covers in the modularity left free; remove all the hole covers added to the composition; duplicate an existing device; replace the device with another; replace the device with a similar one for function; delete selected devices; assign an abbreviation (tag) to the selected device; change the position of the device within the composition.

Through the use of the plug-in it is possible to create logic circuits assigning tags to the objects to be connected (composition, switchboard and utilities) (Fig. 8).

It was decided to implement this function in the plug-in because, during the research and development phase, there were some bugs using the electric connections of Revit MEP. For example, it is not possible to connect a user to multiple command devices (such as a light point controlled by two or more different points). For this reason, it was decided to insert this function of the plug-in into the standard Revit command until Autodesk will implement the Electrical MEP section of Revit.

Once the logical connections have been made, the plug-in allows users to extrapolate the "wiring list", an abacus created ad hoc by plug-ins with all the parameter information that allows for the unequivocal identification of the composition and the utilities connected to it within the project. Without the help of the plug-in, it would be impossible for the user to be able to compose an abacus with the same information, as

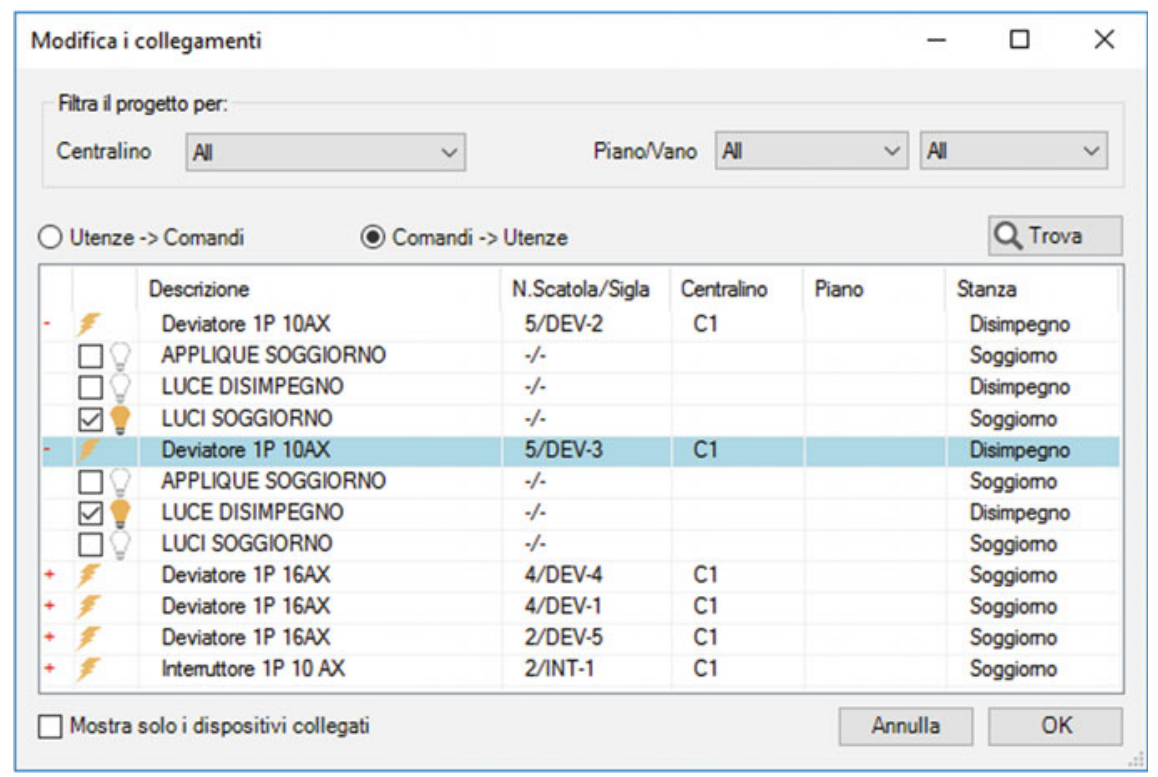

Fig. 8 EasyBIM logic links menu 
they belong to multiple categories of Revit objects. Finally, in the "separate" section of the plug-in you can select different types of lists (reports) to be displayed in Revit in the form of Abachi or that are exportable in XLS format.

The typologies of selectable lists are conceived in order to be able to participate in public procurement (generating items of specifications without reference to the brand and model) either for preliminary and final projects, or to be able to make a calculation for resources (material list).

\section{Conclusion}

Most of The MEP electrical objects currently modeled and available through BIM object libraries satisfy a number of design requirements that are different than those of the Italian national market. The main advantages related to the use of the plug-in are related to the possibility of creating and managing the logical connections (electrical circuits) between the objects and the project users, overcoming the current limitations of the Revit Software.

Moreover, an instantaneous generation of infinite compositions is possible with contained weights (few $\mathrm{KB}$ ) with their informative attributes; there is also the possibility to create both generic objects (without reference to the manufacturer or commercial series) and Vimar objects selectable from the catalogue; along with the automatic assignment of the labels with the respective 2D electrical symbols, with the shape of the frame that varies according to the commercial series; generation and customization of wiring lists by the user; generation of custom lists for schedules and specifications, exportable in Excel; ability to assign parameters (tags) to third-party objects (switchboards and utilities) to insert them into the logic circuits managed by the plug-in; the possibility to set a switchboard as the default switchboard (control: Switchboard current); the ability to extrapolate a set of personalized information (for example, the lists and the wiring list) by means of computer programming in Revit.

Currently, there is no similar instrument on the market capable of instantly composing an electric composition with a low weight (few $\mathrm{KB}$ ), with a 2D graphic production, optimized for the table and the issuance of customizable lists in Revit and in Excel. The plug-in was designed and developed to be able to transpose future implementations of Revit MEP, and especially solve some Autodesk software bugs encountered during this research project.

Some of the implementations of the current commands present in the plug-in have been hypothesized. For example, you could develop a management system of your favourite/recent compositions, to speed up and simplify the design process for the user. Moreover, one of the next objectives is the possibility of managing through an advanced interface the personalization of labels such as the choice of the information to be displayed on the label or which colour to assign according to the type of circuit.

The plug-in, made available on the market in March 2018, includes only part of the Vimar catalogue. The post-launch phase envisages the modelling and informative management of the objects of the Domotic series and the video entry categories. 


\section{References}

BIM: a cosa serve e chi lo userà, Marra A., Edilportale, 2015

BIM: metodi e strumenti, Pavan A., Mirarchi C., Giani M., Tecniche nuove, 2017

BIMForum Specification. Level of development, specification: The guide, Part 1, 2 (2017)

Il BIM e la digitalizzazione dei prodotti, Marra A., Edilportale, 2018

http://www.bimidea.it/

http://www.bebim.it/2018/03/18/standardizzare-gli-oggetti-bim-la-proposta-di-nbs/

https://www.nationalbimlibrary.com/en/nbs-bim-object-standard

http://www.ibimi.it/lod-livello-di-dettaglio-per-il-bim/

https://harpaceas.it/il-bim-e-il-concetto-di-dettaglio-e-lod/

https://www.ingenio-web.it/18667-sistema-dei-lod-italiano-uni-11337-4-2017

https://www.bimandco.com/it/management-lod

https://bimforum.org/lod/

https://www.ingenio-web.it/18926-i-loi-nelle-scale-di-lod-usa-uk-ita

https://store.mepcontent.com/it/product/details/5

https://store.mepcontent.com/it/product/details/44

https://www.gewiss.com/it/it/services/support/softwares/plugin-suite/revit

https://www.vimar.com/it/it/easybim-revit-autodesk-vimar-14955162.html

\section{Standards and Laws}

Level of Development (LOD) Specification: 2019

NBS BIM Object Standard

Norma CEI 64-8:2011 Guida alla Norma CEI 64-8, soluzioni ABB per gli impianti residenziali

Norma CEI 64-8:2007 Norma CEI 64-8 per impianti elettrici utilizzatori

Pass1192-2:2013 Specification for Information Management for the capital/delivery phase of construction projects using Building Information Modelling

UNI 11337-1:2017 Edilizia e opere di ingegneria civile - Gestione digitale dei processi informativi delle costruzioni - Parte 1: Modelli, elaborati e oggetti informativi per prodotti e processi

UNI 11337-4:2017 Edilizia e opere di ingegneria civile - Gestione digitale dei processi informativi delle costruzioni- Parte 4: Evoluzione e sviluppo informativo dei modelli, elaborati e oggetti 
Open Access This chapter is licensed under the terms of the Creative Commons Attribution 4.0 International License (http://creativecommons.org/licenses/by/4.0/), which permits use, sharing, adaptation, distribution and reproduction in any medium or format, as long as you give appropriate credit to the original author(s) and the source, provide a link to the Creative Commons license and indicate if changes were made.

The images or other third party material in this chapter are included in the chapter's Creative Commons license, unless indicated otherwise in a credit line to the material. If material is not included in the chapter's Creative Commons license and your intended use is not permitted by statutory regulation or exceeds the permitted use, you will need to obtain permission directly from the copyright holder.

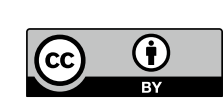

